\title{
Bypass transition in a boundary layer subject to sheet-type freestream disturbance
}

\author{
Kota TOMIUKA*, Yu NISHIO**, Seiichiro IZAWA* and Yu FUKUNISHI* \\ * Department of Mechanical Systems Engineering, Tohoku University \\ 6-6-01 Aramaki-Aoba, Aoba-ku, Sendai, 980-8579, Japan \\ E-mail: izawa@fluid.mech.tohoku.ac.jp \\ ** Department of Systems Design Engineering, Seikei University \\ 3-3-1 Kichijoji-Kitamachi, Musashino-sh, Tokyo, 180-8633, Japan
}

Received: 3 October 2019; Revised: 24 November 2019; Accepted: 5 December 2019

\begin{abstract}
In this study, the receptivity of a flat-plate boundary layer was studied by introducing a thin sheet-type disturbance. An airfoil-shaped device was used to generate a thin disturbance without velocity deficit in which a steady jet was ejected from its trailing edge to the downstream. Despite the absence of strong disturbances in the freestream outside the boundary layer, streaky structures similar to an ordinary bypass transition were generated. They meandered slowly in the spanwise direction where their downstream parts were oscillating in a delayed fashion. Turbulent spots were formed in the further downstream region. Consequently, the energy growth of the low frequency band in the velocity fluctuation spectrum was found to originate from this meandering motion of the streaks, whereas the growth of the middle- and high-frequency bands was attributed to the appearances of the turbulent spots.
\end{abstract}

Keywords : Receptivity, Localized freestream disturbance, Bypass transition, Streaks, Turbulent spot

The boundary layer transition starts from the receptivity process in which the freestream disturbances penetrate into the boundary layer (Saric et al., 2002; Reed et al., 2015). Transition route is then selected depending on the freestream turbulence level $(T u)$ : a Tollmien-Schlichting (TS) wave type transition or a bypass transition. Substantial knowledge regarding the transition process has been accumulated in the several past researches; nonetheless, it remains difficult to predict a transition point accurately even for the orderly TS-type transition whose growth rate can be described well by the linear stability theory. Apparently, this is because the transition point strongly depends on the unpredictable initial amplitude of the disturbance that is taken into the boundary layer (Jay et al., 2010). As such, the relationship between the transition point and the freestream disturbance could be empirically obtained through wind tunnel experiments, and the value of $N$ for the $e^{N}$ method could be given as a function of residual turbulence and sound pressure level in the freestream (Tokugawa et al., 2005). Similarly for the bypass transition, it becomes more difficult to predict the transition point because the linear stability theory is no longer relevant. Westin et al. (1994) pointed out that the onset and the extent of transition differ depending on the qualitative differences of the freestream turbulence characteristics such as the degree of anisotropy. It should be noted that the receptivity process remains an intriguing issue, especially for the bypass transition.

There are several methods that could be employed to add the strong disturbances into the freestream, among which turbulence grids are often used because of their simplicity. Accordingly, these flow disturbances give rise to streamwise elongated regions of high and low streamwise velocities in the boundary layer, or the so-called streaky structures. Their streamwise length increases in proportion to the laminar boundary layer thickness $\delta$ (i.e., proportional to the square root of the downstream distance $x^{1 / 2}$ ) and their energy growth is algebraic, which is close to proportional $x$ (Matsubara and Alfredsson, 2002). Moreover, the initial disturbance energy in the boundary layer is proportional to $T u^{2}$, whereas the transitional Reynolds number based on the downstream distance varies as $T u^{-2}$ (Fransson et al., 2005). When the plate has a blunt leading edge, the receptivity becomes higher at the nose section and the transition takes place considerably earlier than the sharp plate. Moreover, when the leading edge is blunt, the transition position is almost insensitive to $T u$ unlike the sharp leading edge case, though both transition mechanisms are considered to possess the same algebraic growth of the low frequency fluctuations (Zhigulev et al., 2009). Nonetheless, in these experiments, it is difficult to clarify the 
origin of velocity fluctuations in the boundary layer, because the disturbance that has been provided by the turbulence grid contaminates the entire flow field, including the freestream just outside the boundary layer. As such, the disturbances in the freestream might either disturb the flow inside the boundary layer directly or influence indirectly through the vorticity creation at the wall.

The leading edge receptivity does not only depend on the geometry of the leading edge, but also on the nature of the disturbances. Regardless of the nose bluntness, the boundary layer is most receptive to freestream streamwise vortices with zero frequency, which has an ability to trigger the formation of long streaks (Schrader et al., 2010; Nagarajan et al., 2007). Boiko et al. (2002) observed that a single streak is generated in response to an artificially introduced axial vortex in the freestream outside the boundary layer using the tip vortex of a micro wing. Freestream vortices normal to the plate can also excite streaky structures that are elongated in the spanwise direction. Essentially, an oncoming vortex having its axis normal to the plate is converted into a longitudinal vortex inside the boundary layer when it hits the leading edge. Schrader et al. (2010) suggested that the stretching and tilting of the wall-normal vorticity component are responsible for the generation of streaky structures. Nishio et al. (2013) found later on that the streamwise streaky structures can be directly generated by the strong induced velocities of the wall-normal vortices outside the boundary layer, instead of longitudinal vortex structures in the near-wall region. Nagarajan et al. (2007) argued the effect of the nose bluntness on the bypass transition imposing a homogeneous isotropic turbulence at the inflow boundary.

The appearance and growth of streamwise streaks have been considered to play an important role in bypass transition. Theoretically, this process can be interpreted as a sequence of events from a transient growth of streaky structures to a secondary instability, such as streak instability. The transient growth, which is characterized by an algebraic growth in space and time followed by an exponential decay due to the viscosity (Reshotko, 2001), arises through the non-orthogonal nature of the Orr-Sommerfeld and the Squire modes. If the streak amplitude reaches a certain threshold during the first algebraic growth, their inflectional velocity profiles of low-speed streaks in both the wall-normal and spanwise direction become sensitive to time-dependent disturbance, which is the secondary instability (Elofsson et al., 1999; Asai et al., 2007). The transition is generally triggered by a breakdown of laterally oscillating streaks, which arises from the instability of the spanwise profile, or the so-called sinuous instability, and not the varicose instability (Andersson et al., 2001). However, the streak breakdown is apparently not a unique process. In fact, several breakdown scenarios have been proposed through numerical and experimental investigations. Jacobs and Durbin (2001) pointed out that the streak breakdown is initiated when the near-wall streaks are lifted up toward the boundary layer edge by low-frequency freestream disturbances, and then become receptive to high-frequency freestream disturbances. Schlatter et al. (2008) showed that the streak instability is responsible for the generation of turbulent spots. Cherubini et al. (2014) provided another scenario in which they demonstrated that for a high $T u$ with large integral scale, the transition is initiated by the occurrence of localized vortical structures, which are directly generated in the boundary layer by the freestream disturbance.

The present experimental study aims to provide a more comprehensive explanation on the receptivity of a boundary layer on a flat plate with an elliptic leading edge under the condition that a strong disturbance is applied only onto the leading edge of the plate. An airfoil-shaped disturbance generator is designed to introduce a sheet-type disturbance without velocity deficit, having a thickness that is one-fifth of the plate thickness. The generator is installed along the extension of the middle of thickness of the downstream plate so that the disturbance hits the stagnation line of the plate. Subsequently, the bypass transition process is observed in detail by focusing on the development of the streak and the generation of turbulent spots.

\section{Experimental setup}

The experiments were conducted in a blowout-type wind tunnel whose settling chamber had a square cross section with a side length of $1500 \mathrm{~mm}$ and a nozzle with a contraction ratio of 9: 1. The test section measured $2000 \mathrm{~mm}$ in the streamwise direction, and $500 \mathrm{~mm}$ in both the vertical and spanwise directions. The tunnel had an adjustable ceiling plate, which regulates the pressure distribution in the streamwise direction. A schematic view of the experimental setup is displayed in Fig. 1. A flat plate made of aluminum alloy was horizontally mounted $200 \mathrm{~mm}$ above the bottom surface of the test section in order to avoid the boundary layer from developing along the inner walls of the test section. The plate was 1610-mm long, 490-mm wide, and 50-mm thick; moreover, it had a semi-elliptical leading edge with 12:1 aspect ratio, i.e., $300 \mathrm{~mm}$ in length. A 190-mm long flap was mounted at the trailing edge of the flat plate in order to adjust the stagnation point and prevent a flow separation at the leading edge. The $x$ - and $z$-axes were in the streamwise and spanwise directions, respectively, and their origin was set at the center of the tip of the leading edge, whereas the $y$-axis 


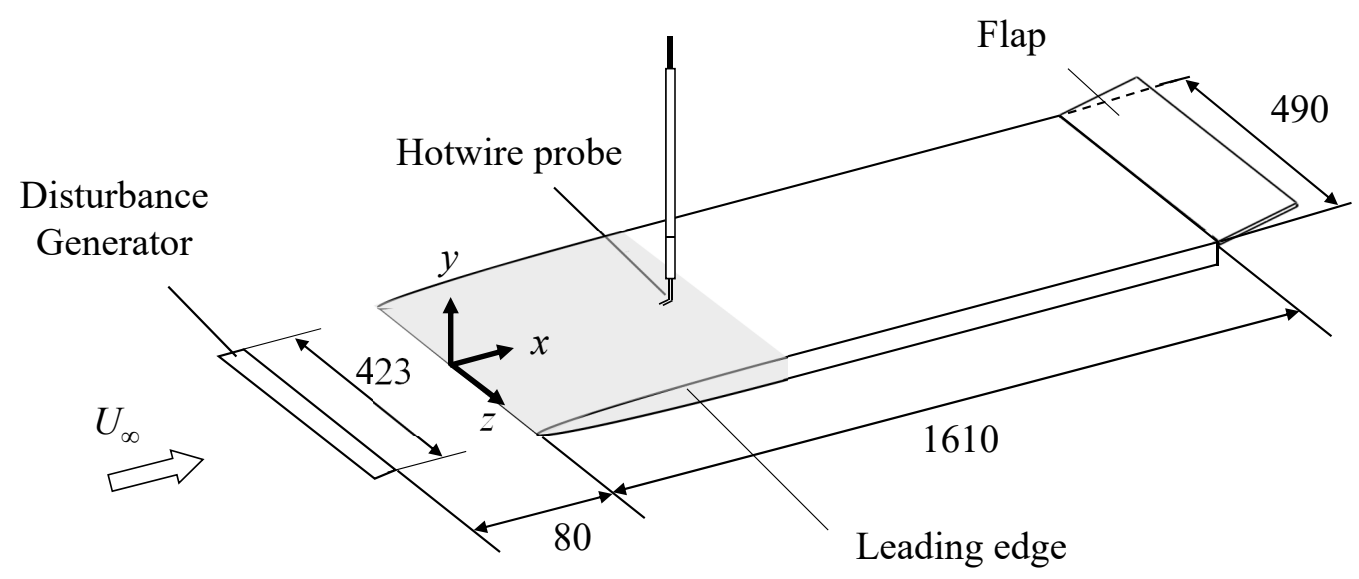

Fig. 1 Schematic view of the experimental setup.

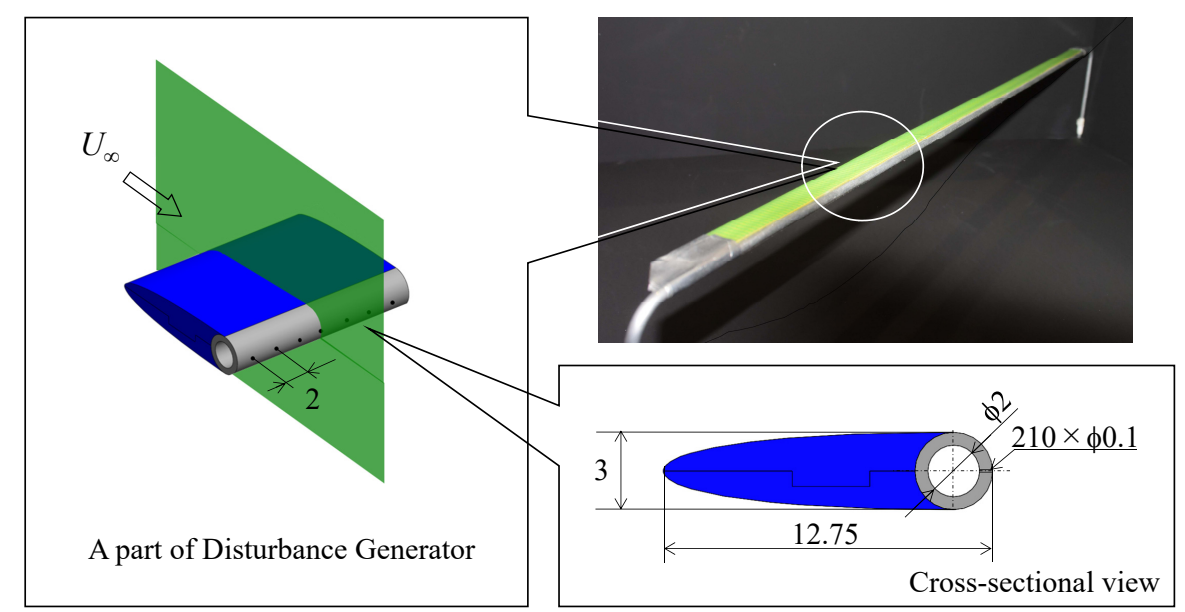

Fig. 2 An airfoil-shaped disturbance generator.

was orthogonal to both with the locations of $y=0 \mathrm{~mm}$ always at the wall surface for $x=0$, and same as the tip of the leading edge for $x<=0$. The freestream velocity $U_{\infty}$ was fixed at $8 \mathrm{~m} / \mathrm{s}$, and the freestream intensity was less than $0.3 \%$ of $U_{\infty}$. Variations in pressure coefficients $C_{p}$ in the test section were adjusted to be less than $1 \%$, so that the pressure gradient in the streamwise direction could be neglected.

A disturbance generator, whose configuration appears in Fig. 2, was mounted 80-mm upstream of the leading edge at $y=0 \mathrm{~mm}$. The generator had a thickness of $3 \mathrm{~mm}$, chord length of $12.75 \mathrm{~mm}$, and width of $423 \mathrm{~mm}$. It consisted of two parts, namely, an airfoil-shaped body made of ABS resin and an aluminum pipe with an inner diameter of $2 \mathrm{~mm}$. The airfoil-shaped body assumed a model NACA0008 configuration, based on the shape produced by the Fused Deposition Modeling 3D printer (Dimension, BST768) of the Creative Engineering Center of Tohoku University. The pipe had 210 small holes with 0.1 -mm diameters, which face the downstream side. The holes were opened every 2 mm in the spanwise direction. The air was supplied from an air compressor (ANEST IWATA, HX4004) through one end of the pipe, while the opposite end was closed. The flow rate of the compressed air was controlled by a regulator (Monotaro, FR-02), so that the steady jet ejected toward downstream would compensate the velocity deficit of the distance generator's own wake. Therefore, local velocity fluctuations could be introduced into the flow while keeping the velocity distribution uniform. A sheet jet had a total width of $418 \mathrm{~mm}$, which corresponds to approximately $87.1 \%$ of the spanwise width of the flat plate.

A single hot-wire probe, supported by a three-dimensional traversing mechanism, was used for the velocity measurement. The probe was connected to a constant temperature anemometry (CTA) unit. Several fixed probes were also used for simultaneous measurements at two or three points. Signals from the hot-wires were smoothed by fourth-order Butterworth-type low-pass filters with a cut-off frequency of $2.5 \mathrm{kHz}$ to prevent aliasing errors. The filtered signals were 
(a)

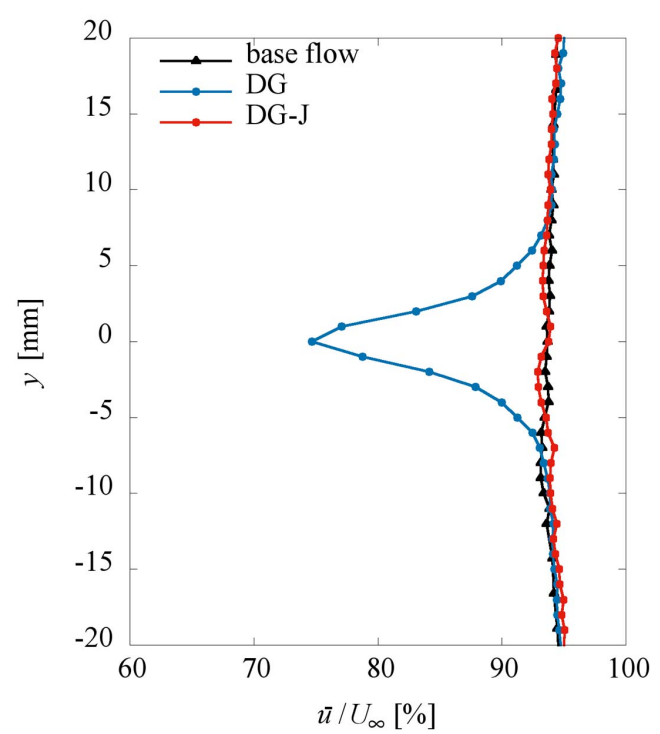

(b)

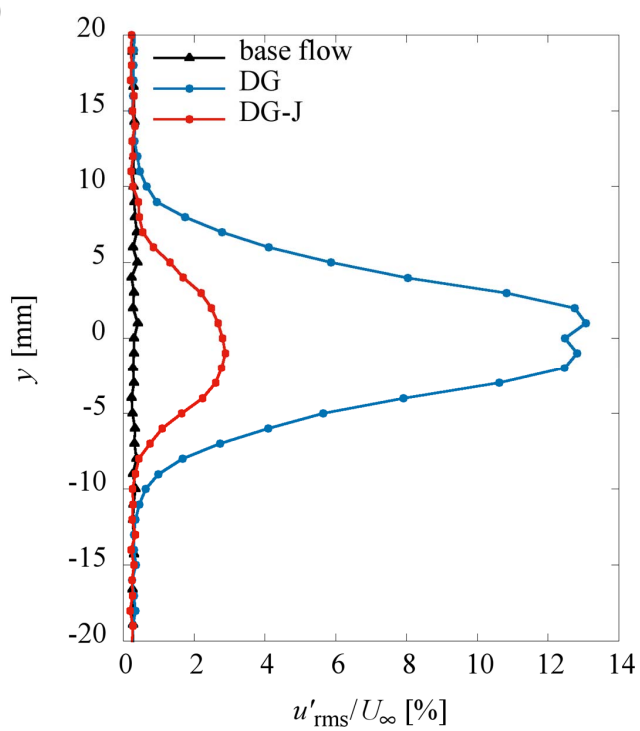

Fig. 3 Profiles of (a) $\bar{u} / U_{\infty}$ and (b) $u_{\mathrm{rms}}^{\prime} / U_{\infty}$ at $x=-50$ and $z=0 \mathrm{~mm}$. The black line indicates the base flow without disturbance generator (DG); the blue line indicates the result when the DG is installed in the freestream and; the red lines indicates the case where additionally, the jet is ejected from the trailing edge of DG. The velocity deficit disappears as a result of the jet ejection, and a sheet-type disturbance is introduced into the freestream.

then converted to series of digital data using a 16-bit analog-digital (A/D) converter (Interface, PCI-3155) at the sampling rate of $5 \mathrm{kHz}$. The number of data for one series sampling was $2^{14}$.

When the disturbance generator was not installed in the freestream, the mean velocity profile of a boundary layer developing on the plate surface, i.e., the base flow, agreed well with the Blasius profile, which is a self-similar profile of the zero-pressure gradient flat-plate boundary layer. In fact, the Blasius profile was observed at both the leading edge section $(x \leqq 300 \mathrm{~mm})$ and the flat plate section $(300 \mathrm{~mm} \leqq x)$, which were connected at $x=300 \mathrm{~mm}$. Judging from the velocity profiles on the flat plate section, the virtual origin was located approximately $110 \mathrm{~mm}$ upstream of the leading edge. The root-mean square (RMS) values of the velocity fluctuations were less than $1 \%$ of the freestream velocity, regardless of the streamwise and spanwise stations.

\section{Results and discussion}

\subsection{Characteristics of introduced disturbance}

The characteristics of the disturbance that was added to the freestream using the airfoil-shaped device introduced herein were examined by comparing two cases with and without jet ejection from its trailing edge. Figure 3 shows a comparison of the wall-normal profiles of the time-averaged velocity $\bar{u} / U_{\infty}$ and RMS fluctuation $u_{\mathrm{rms}}^{\prime} / U_{\infty}$ at $z=0 \mathrm{~mm}$ and $x=-50 \mathrm{~mm}$, which was 30-mm downstream of the disturbance generator. Three cases- no disturbance generator (base flow), disturbance generator installed but not activated (DG), and disturbance generator ejecting jet (DG-J) - are shown in the figure. A velocity deficit was observed in the wake of the disturbance generator in the region of $y=-7$ to $7 \mathrm{~mm}$, and the RMS profile of the velocity fluctuations showed two peaks, which reached a maximum of $12-14 \%$ of the freestream velocity. A fast Fourier transform (FFT) analysis of the result revealed that the velocity spectrum at $y=0 \mathrm{~mm}$ possessed a strong peak at approximately $500 \mathrm{~Hz}$, close to the frequency of the typical Karman vortex street, which is estimated as $\left.533 \mathrm{~Hz}(=0.2 \times 8.0) /\left(3.0 \times 10^{-3}\right)\right)$, thereby elucidating the cause of the two peaks in the RMS profile. With this aside, when the jet was ejected in order to compensate the velocity deficit, the velocity profile became almost flat, and close to the base flow. Moreover, the two peaks in the RMS profile disappeared because of the absence of the Karman vortex street; the maximum RMS value also got reduced to about $3 \%$ of $U_{\infty}$ at $z=0 \mathrm{~mm}$, within which the half-width of the RMS profile was approximately $10 \mathrm{~mm}$ in the $y$ direction. Considering that the plate thickness was $50 \mathrm{~mm}$, this implied that the relative thickness of this sheet-type disturbance was thin, that is, only one-fifth of the plate thickness.

Accordingly, Fig. 4 shows the spanwise variations of $\bar{u} / U_{\infty}$ and $u_{\mathrm{rms}}^{\prime} / U_{\infty}$ behind the generator at $y=0.0 \mathrm{~mm}$, for three streamwise stations, $x=-50,-30$, and $-15 \mathrm{~mm}$, downstream of the disturbance generator and upstream of the leading edge of the flat plate. Here, the streamwise velocity was observed to decrease gradually while approaching the stagnation 
(a)

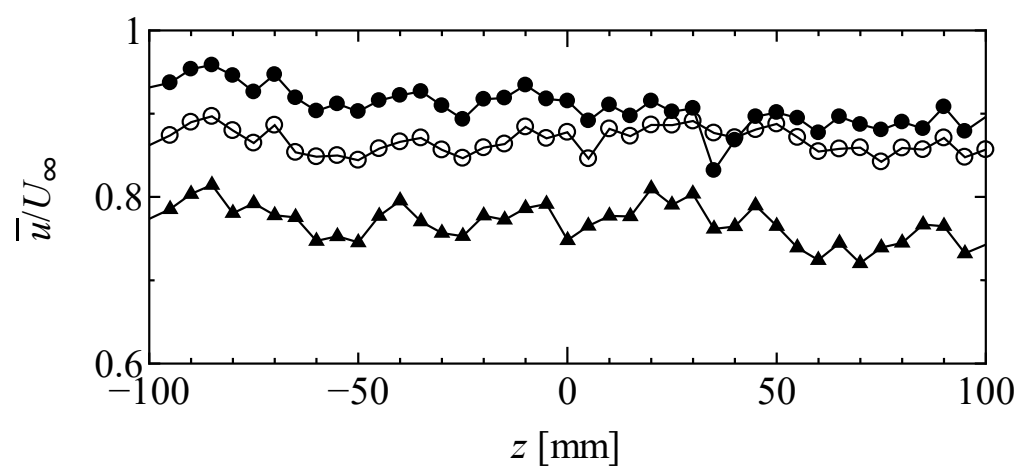

(b)

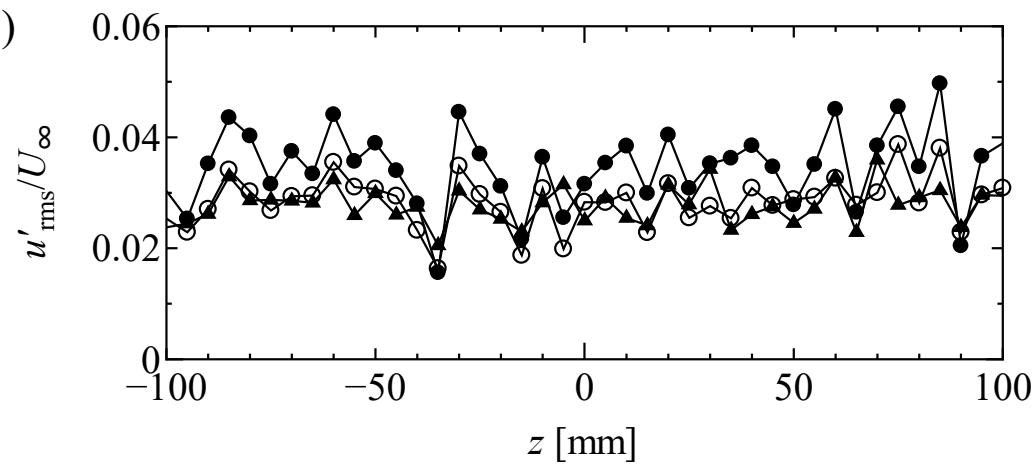

Fig. 4 Spanwise profiles of (a) $\bar{u} / U_{\infty}$ and (b) $u_{\mathrm{rms}}^{\prime} / U_{\infty}$ at $y=0 \mathrm{~mm}$. Closed circle, $x=-50 \mathrm{~mm}$; Open circle, $x=$ $-30 \mathrm{~mm}$; Closed triangle, $x=-15 \mathrm{~mm}$. The disturbance introduced into the freestream is almost uniform. On average, $u_{\mathrm{rms}}^{\prime}$ is $2.3 \%$ of $U_{\infty}$ at $x=-15 \mathrm{~mm}$.
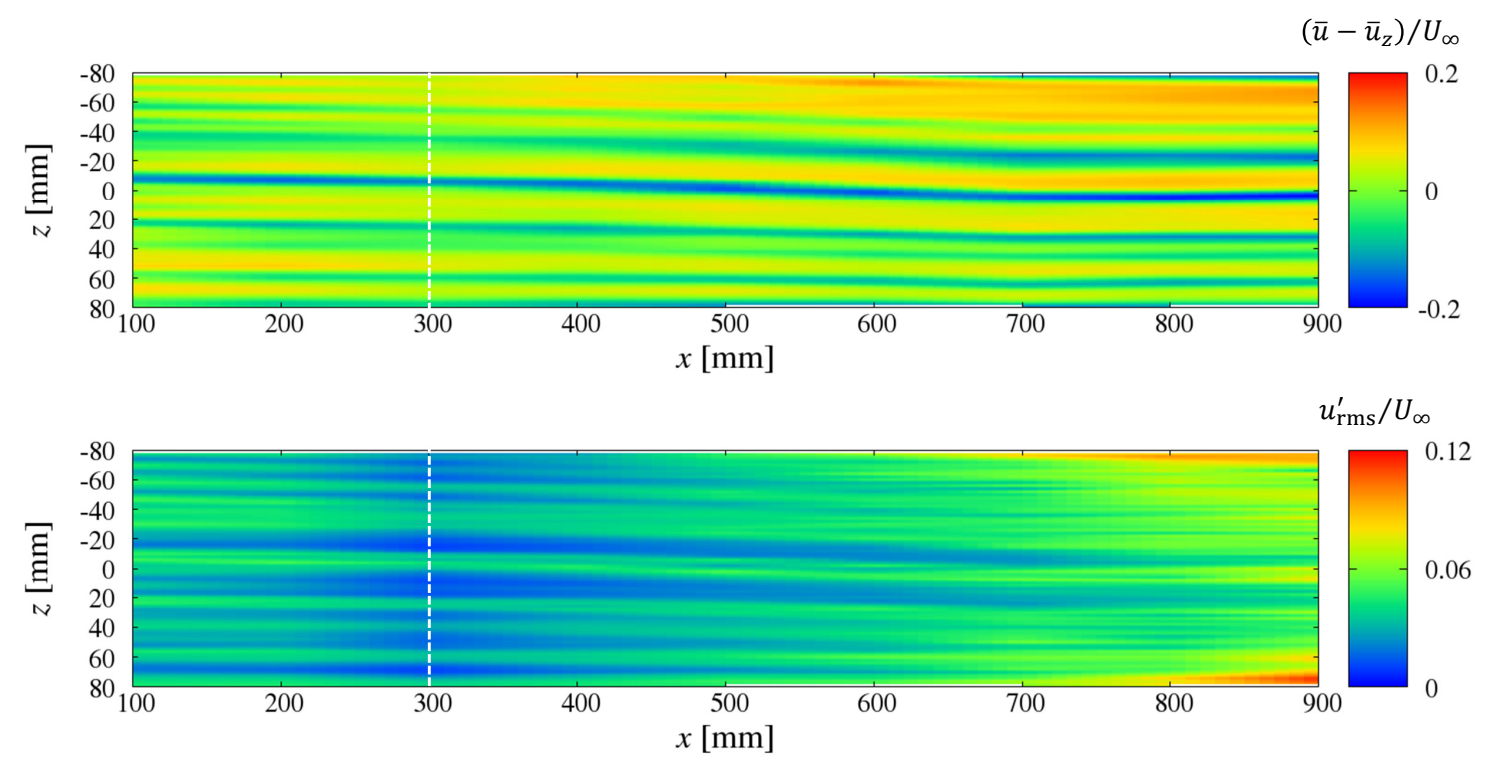

Fig. 5 Contour maps of (a) $\left(\bar{u}-\bar{u}_{z}\right) / U_{\infty}$ and (b) $u_{\mathrm{rms}}^{\prime} / U_{\infty}$ in the $x z$ plane at $\eta=3$. Broken line at $x=300 \mathrm{~mm}$ indicates the juncture. Stationary streaky structures are observed throughout the boundary layer. 


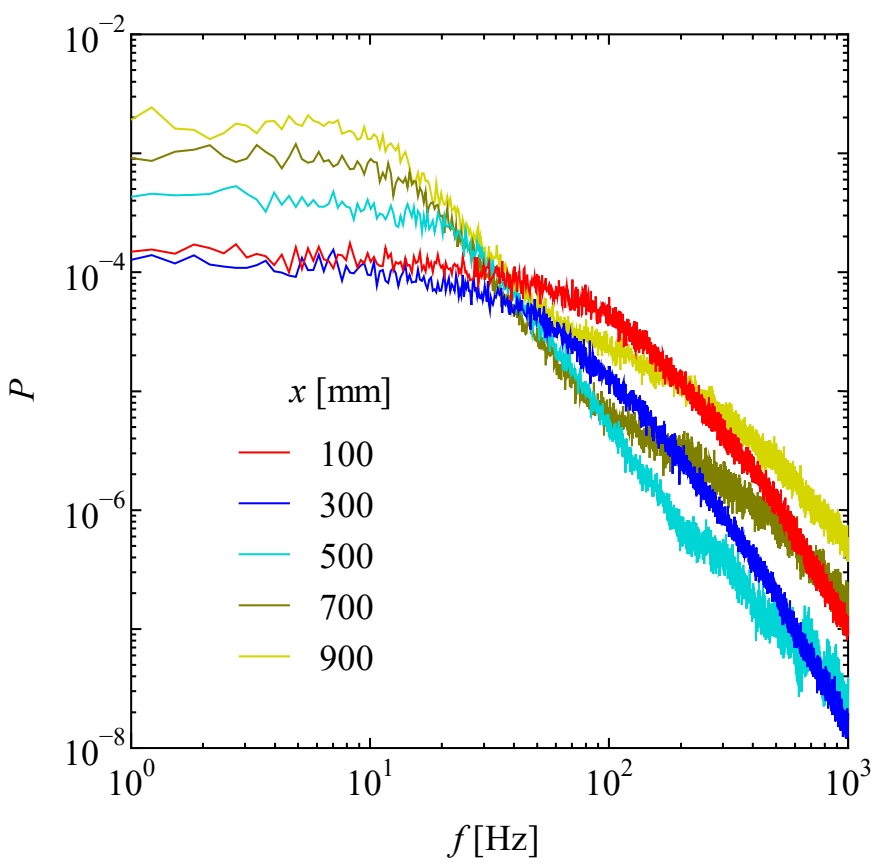

Fig. 6 Spanwise-averaged power spectra of $u^{\prime}$ at $\eta=3$.

point of the plate, which reflects the adverse pressure gradient. Apparently, it also descended slightly in the positive spanwise direction, mainly because of the unevenness of the jet velocity ejecting from the trailing edge of the disturbance generator. Meanwhile, the ejection velocity on the closed end of the pipe in the negative $z$ direction tended to be faster than that on the open end in the positive $z$ direction that was connected to an air compressor. This tendency agrees with the theoretical and experimental works (Toda et al., 2019). As such, the authors of the study assumed the spanwise difference to be negligible. Moreover, the spanwise dispersion of $\bar{u} / U_{\infty}$ at $x=-15 \mathrm{~mm}$ was fluctuating quite uniformly within a small range of $2.3 \%$ of the freestream velocity. The variation of $u_{\mathrm{rms}}^{\prime} / U_{\infty}$ also decreased near the stagnation point. As such, the relation between the spanwise variations of the mean velocity $\bar{u} / U_{\infty}$ and the velocity fluctuation $u_{\mathrm{rms}}^{\prime} / U_{\infty}$ could not be clearly observed. Nonetheless, it is worth noting that the value of $u_{\mathrm{rms}}^{\prime} / U_{\infty}$ maintained a relatively high value, on a spanwise average of $2.8 \%$ at $x=-15 \mathrm{~mm}$, which demonstrates that the disturbance generator was able to introduce a sheet-type, uniform disturbance into the freestream without producing a velocity deficit.

\subsection{Generation and growth of streaky structures}

In order to investigate the response of the boundary layer to the sheet-type disturbance, the velocity fluctuations were measured in the $x z$ plane of $\eta\left(=\sqrt{U_{\infty} / v x}\right)=3$. Figure 5(a) shows the contour maps of the spanwise fluctuation $\left(\bar{u}-\bar{u}_{z}\right) / U_{\infty}$, where $\bar{u}_{z}$ is the spanwise-averaged velocity of $\bar{u}$ at each streamwise station. Measurement intervals are $100 \mathrm{~mm}$ in the streamwise direction and $2 \mathrm{~mm}$ in the spanwise direction. The broken line at $x=300 \mathrm{~mm}$ indicates the location of the junction between the elliptic leading edge part and flat plate part. Stationary high- and low-velocity regions elongated in the streamwise direction and alternately arranged in the spanwise direction were present. The boundary layer was in a laminar state even at $x=900 \mathrm{~mm}$, which was the most downstream location in the measurement. In this paper, the streaky structures were judged by the streamwise velocities that were measured by the height of $\eta=3$. Apparently, the corresponding low-speed regions were narrow and were more concentrated than the high-speed regions, especially in the downstream region. Comparing Figs. 4(a) and 5(a), these low-speed locations could be observed right downstream of the locally low time-averaged velocities in the sheet-type disturbance generated by the DG. The result suggests that the low-speed streaks originated from the small spanwise variation of time-averaged local velocities behind the disturbance generator.

A similar elongated pattern could be seen in the map of RMS fluctuation $u_{\mathrm{rms}}^{\prime} / U_{\infty}$ (Fig. 5(b)). The RMS fluctuations tended to be stronger at the low-speed streak locations, and increased gradually downstream. Likewise, note that the spanwise ends of the flow field, within $x=700-900 \mathrm{~mm}$ and $|z|>70 \mathrm{~mm}$, were contaminated by the sidewall boundary layers and the generated tip vortices at the spanwise edges of the disturbance generator upstream. Thus, only those measurement results within the range of $z= \pm 70 \mathrm{~mm}$ were employed herein. 


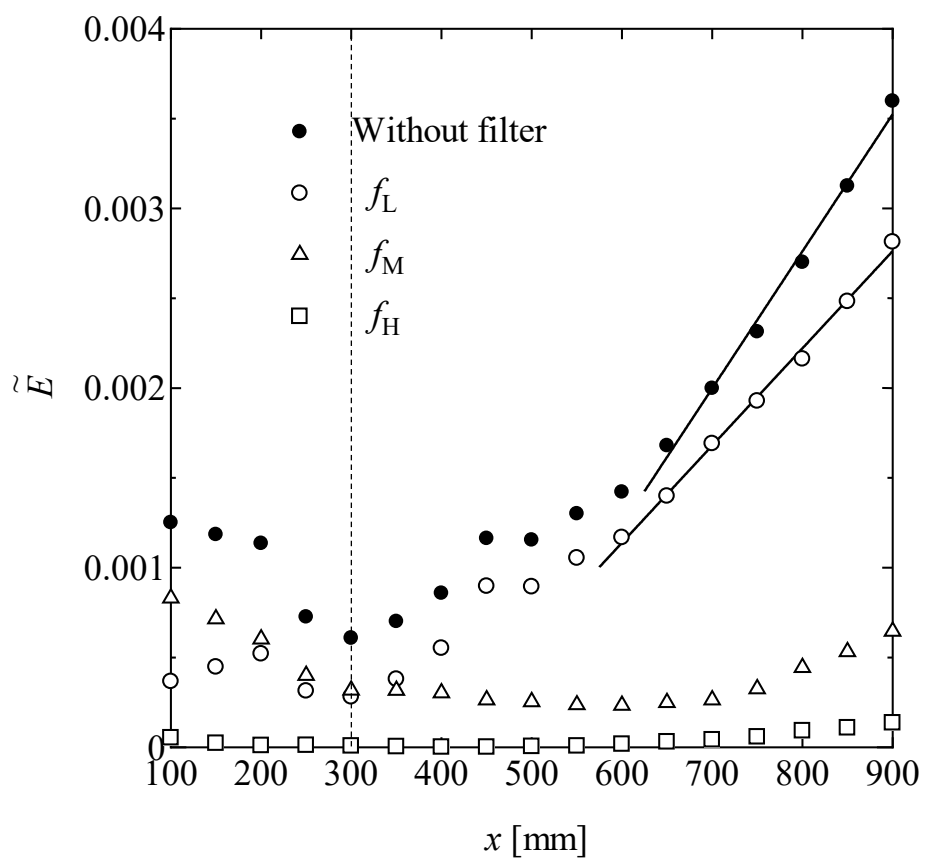

Fig. 7 Spanwise-averaged energy of $u^{\prime}$ at $\eta=3$. The broken line at $x=300 \mathrm{~mm}$ indicates the juncture. The solid lines are approximated straight lines for the total and low-frequency components $f_{\mathrm{L}}$ ( $<30 \mathrm{~Hz}$ ), with inclinations of $7.63 \times 10^{6}$ or $5.41 \times 10^{-6}$.

Figure 6 shows the power spectra of the spanwise-averaged velocity fluctuation $P$ at $\eta=3$ for each streamwise station. The total energy, which could be obtained as the integral value of a power spectrum, decreased within the nose section, $x \leq 300 \mathrm{~mm}$, especially for high-frequency components higher than $40 \mathrm{~Hz}$. Downstream of the juncture, low-frequency components lower than $30 \mathrm{~Hz}$ started gaining energy. Further, after $x=500 \mathrm{~mm}$, high-frequency components higher than a couple of hundred $\mathrm{Hz}$ began increasing their energy again. In order to provide a more quantitative observation of the growth of the fluctuations, the frequency components were divided into three bands as follows:

(1) low component $\left(f_{\mathrm{L}}: 0<f \leq 30 \mathrm{~Hz}\right)$;

(2) middle component $\left(f_{\mathrm{M}}: 30 \mathrm{~Hz}<f \leq 300 \mathrm{~Hz}\right)$ and;

(3) high component $\left(f_{\mathrm{H}}: 300 \mathrm{~Hz}<f\right)$.

Additionally, their spanwise-averaged energies $\tilde{E}$ were obtained along the streamwise direction at $\eta=3$. Here, the value of $\tilde{E}$ is given by

$$
\tilde{E}=\frac{1}{140} \int_{-70}^{70}\left(\frac{u_{\mathrm{f}, \mathrm{rms}}^{\prime}}{U_{\infty}}\right)^{2} d z
$$

where $u_{\mathrm{f}, \mathrm{rms}}^{\prime}$ is the RMS of filtered velocity fluctuation, $f_{\mathrm{L}}(<30 \mathrm{~Hz}), f_{\mathrm{M}}$, and $f_{\mathrm{H}}(>300 \mathrm{~Hz})$.

A plot of the streamwise variation of each spectral band is displayed in Fig. 7. The total energy is also shown in the figure for comparison. Here, the broken line represents the location of the juncture between the curved surface of the leading edge and the flat plate. All the frequency components tended to be suppressed in the leading edge, although their behaviors in the downstream flat plate section were different, depending on the frequency bands. Based on the figure, the lowest frequency components, which were lower than $30 \mathrm{~Hz}$, began increasing right after the juncture. Their growth rate became constant in the region downstream of $x=650 \mathrm{~mm}$, which was approximately $5.41 \times 10^{-6}$. The middle and high frequency components, on the other hand, decayed continuously up to $x=600 \mathrm{~mm}$ and $450 \mathrm{~mm}$, respectively, and their growths after $x=650 \mathrm{~mm}$ became gradual. The middle-frequency components, which were predominant in the nose section, exhibited only a small growth in the flat plate region, whereas the high-frequency components remained quite small in the entire region. Consequently, the low-frequency components below $30 \mathrm{~Hz}$ demonstrated a dominant contribution on the total energy growth having a gradient of $7.63 \times 10^{-6}$. Asai et al. (2007) likewise observed the growth of the low-frequency fluctuations for the turbulent boundary layer in which the near-wall fluctuations were significantly reduced by the local suction. They were able to observe the growth of higher-frequency disturbances after the sinuous instability resulting from the sufficiently intensified low-speed streaks became dominant. Therefore, the following experiment was carried out in order to find the cause of the growth of the low-frequency components. 


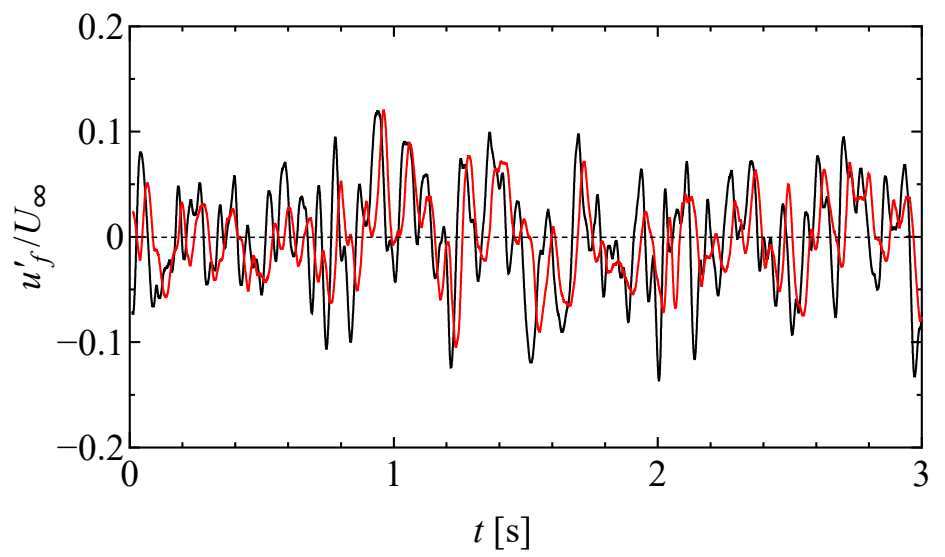

Fig. 8 Sample filtered signals of $u^{\prime} / U_{\infty}$ at $z=20 \mathrm{~mm}$. Black line, $x=650 \mathrm{~mm}$ and $\eta=4$; red line, $x=700 \mathrm{~mm}$, and $\eta=2.5$.

(a)

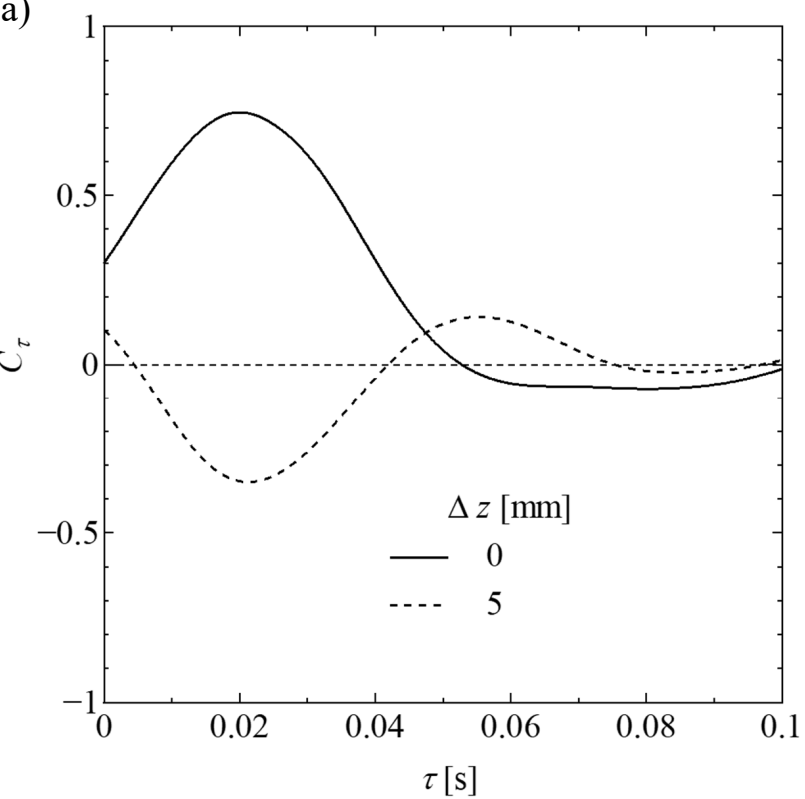

(b)

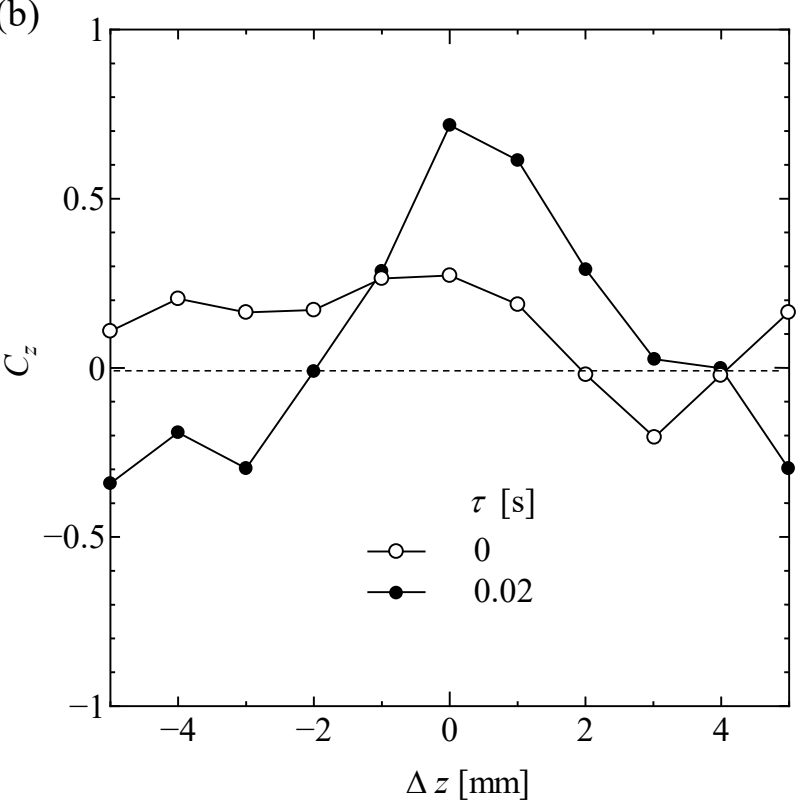

Fig. 9 Correlation coefficients of $u^{\prime}$. (a) Temporal distributions of the correlation coefficients for $\Delta z=0$ - and 5-mm cases; (b) Spanwise distributions for $\tau=0$ - and 0.02 -s cases when $\Delta z=0 \mathrm{~mm}$.

The velocity fluctuations were measured simultaneously at two different streamwise stations, in particular, at $x=$ 650 and $700 \mathrm{~mm}$. An upstream probe at $\eta=4$ was moved in the spanwise direction, while a reference probe downstream was fixed at $\eta=2.5$ and $z=-20 \mathrm{~mm}$. Both probes were slightly shifted toward the wall-normal direction to avoid the interference between the probes. In addition, a reference spanwise station of $z=-20 \mathrm{~mm}$ was placed between the highand low-speed streaks where the spanwise velocity gradient was largest (Fig. 5), so that the meandering motion of the streaks could be detected. The high-frequency components were then filtered out in advance from the probe signals by applying a 101-point moving average. As such, the cut-off frequency $f_{c}$ given by

$$
f_{c}=\frac{0.4429}{\sqrt{N^{2}-1}} f_{s}
$$

was approximately $22 \mathrm{~Hz}$. Here, $N$ is the length of moving average, and $f_{s}(=5 \mathrm{kHz})$ is the sampling frequency.

Figure 8 illustrates examples of the filtered fluctuation signals $u_{f}^{\prime}$. Note that both probes were aligned at the same spanwise location, $z=-20 \mathrm{~mm}$, where the downstream probe was set near the wall to avoid the influence of the upstream probe's wake. The two waveforms were quite similar to each other, except that the signal from the downstream probe 


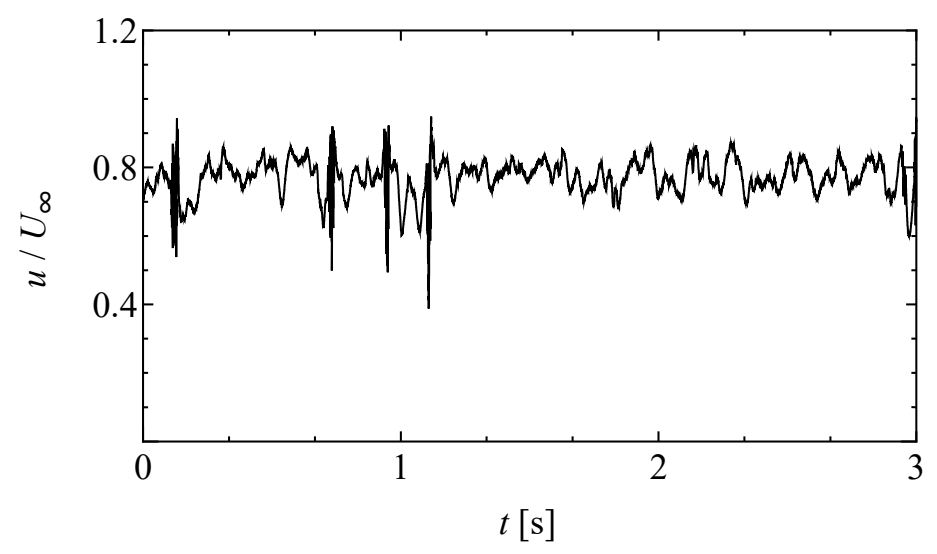

Fig. 10 Raw velocity signal of $u^{\prime}$ at $x=800 \mathrm{~mm}, \eta=3$, and $z=0 \mathrm{~mm}$.
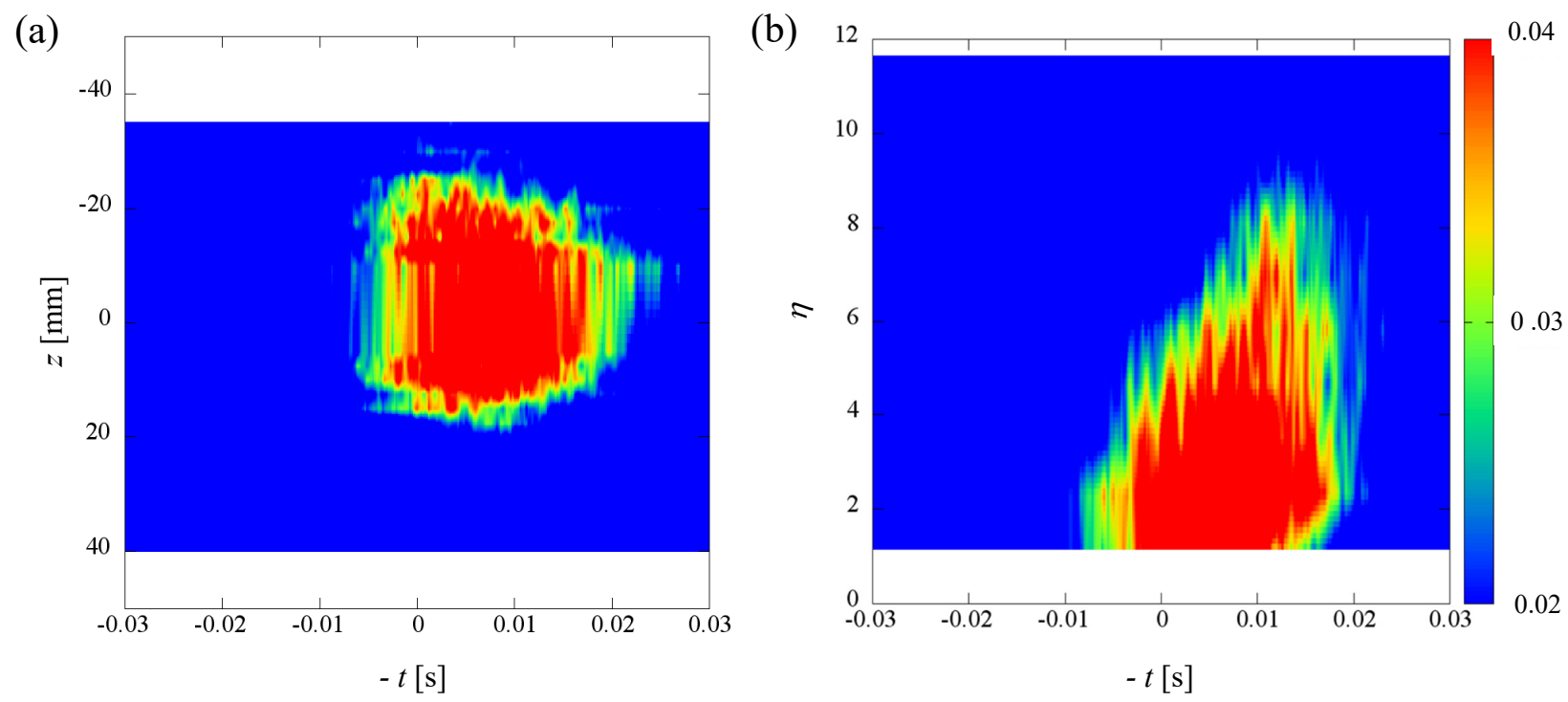

Fig. 11 Fluid structure responsible for the spike waveform. The contour maps represent the ensemble-averaged intensity of high-frequency components that have been normalized by the freestream velocity at $x=800$ $\mathrm{mm}$ in (a) the $z t$ plane at $\eta=3$ (a) and (b) the $\eta t$ plane at $z=5 \mathrm{~mm}$.

was slightly delayed and weaker. In Fig. 9 (a), the solid line represents the temporal correlation functions $C_{\tau}$ of these two signals. More specifically, the correlation is given by

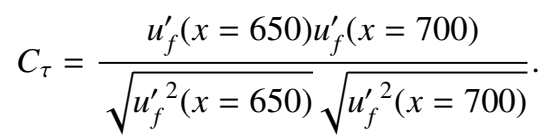

where $\Delta z$ indicates the relative spanwise distance from the downstream reference probe at $z=-20 \mathrm{~mm}$. The value of $C_{\tau}$ reached a maximum of 0.75 at $\tau=0.02 \mathrm{~s}$, after which it decreased to zero. The correlation profile of a neighboring high-speed streak displayed a similar, but opposite trend, at around $z=-15 \mathrm{~mm}(=-20+\Delta z)$, which gave a minimum value at the same timing $\tau=0.02 \mathrm{~s}$.

Another measurement was made by moving the upstream probe in the spanwise direction in order to measure the spatial correlations. The result is shown in Fig. 9 (b). When the time delay $\tau$ was changed from 0 to $0.02 \mathrm{~s}$, the spatial correlation $C_{z}$ became larger and reached the maximum at around $\Delta z=0 \mathrm{~mm}$. It should be noted that the high correlation region was limited to within $-1<\Delta z<1 \mathrm{~mm}$, which was quite narrow compared to the streamwise length of the flow structure. These space-time correlation results could be rationally explained as the meandering of the streaky, snake-like structures in the spanwise direction. And, from the local averaged velocity at the upstream probe location, i.e. $\bar{u}=6.3$ $\mathrm{m} / \mathrm{s}$, and the maximum/minimum time delay of $\tau=0.02 \mathrm{~s}$, the half wavelength of the snake-like structure was obtained 


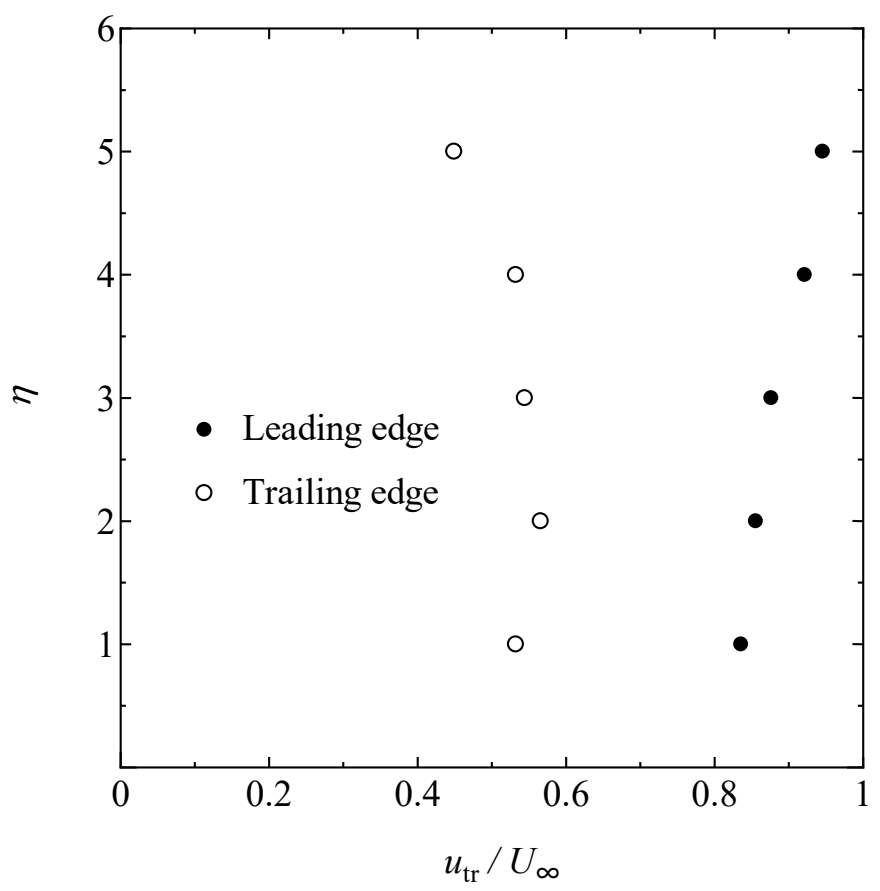

Fig. 12 Traveling speeds of the leading and trailing edges of the flow structure detected at $z=0 \mathrm{~mm}$ for $x=700$ $-800 \mathrm{~mm}$.

as approximately $126 \mathrm{~mm}$. This meandering motion would result in the energy growth of the low-frequency bands in the energy spectrum. The probable cause of the meandering is the sinuous instability of the streaks, which indicate that the low-speed streaks was intensified enough to become unstable.

\subsection{Breakdown of streaky structures}

As shown in Fig. 10, downstream of $x=400 \mathrm{~mm}$, spiky signals could be intermittently seen in the raw data; further, the number of spikes increased monotonically in the streamwise direction from around $x=450 \mathrm{~mm}$. In order to identify the flow structure generating the spike waveform, simultaneous three-point measurement was performed at $x=800 \mathrm{~mm}$ and $\eta=1$ using two fixed probes and one movable probe. The fixed probe at $z=0 \mathrm{~mm}$ (hereafter, the reference probe) was used for spike detection. The other fixed probe (hereafter, the contamination probe) at $z=-50$ or $50 \mathrm{~mm}$ depending on the spanwise location of the movable probe, was used to check whether the spike detection was caused by contamination due to the sidewall boundary layers. The velocity data from the movable probe, which were obtained at every $2.5 \mathrm{~mm}$ in the spanwise direction, were analyzed using a combination of a conditional sampling technique and an ensemble averaging technique. Nonetheless, note that when the velocity was measured at $z=0 \mathrm{~mm}$, the data from the reference probe itself were used, instead of the data from the movable probe. In each location, the number of sampling data was $2^{16}-2^{20}$, to secure 60 - 70 samples. The detailed process is described as follows:

(1) The high-frequency components were removed by filtering the raw data from each probe through the application of a 21-point moving average with a cut-off frequency of $105.6 \mathrm{~Hz}$.

( 2 ) The period when the smoothed reference signal exceeded the threshold value of $u^{\prime} / U_{\infty}=0.2$ was recognized as a spike candidate.

(3) If the smoothed signal from the contamination probe in this period was lower than $u^{\prime} / U_{\infty}=0.1$, then the effect of sidewall contamination was judged as negligible.

(4) The reference time was defined as the elapsed time from the center of each wave of the spike.

( 5 ) The high-frequency components were obtained by subtracting the smoothed sampled signal from the raw signal.

( 6 ) Their absolute values were ensemble-averaged based on each spike detection.

Figure 11 shows the contour maps of the ensemble-averaged intensity of the high-frequency components that have been normalized by the freestream velocity at $x=800 \mathrm{~mm}$ in the $z t$ plane at $\eta=3$ and the $\eta t$ plane at $z=5 \mathrm{~mm}$. A strongly-disturbed region, which can be assumed to be turbulent, could be seen spreading in both the spanwise and wallnormal directions; its spanwise width was approximately $40 \mathrm{~mm}$. Additionally, it had a sharp leading edge that reached a 


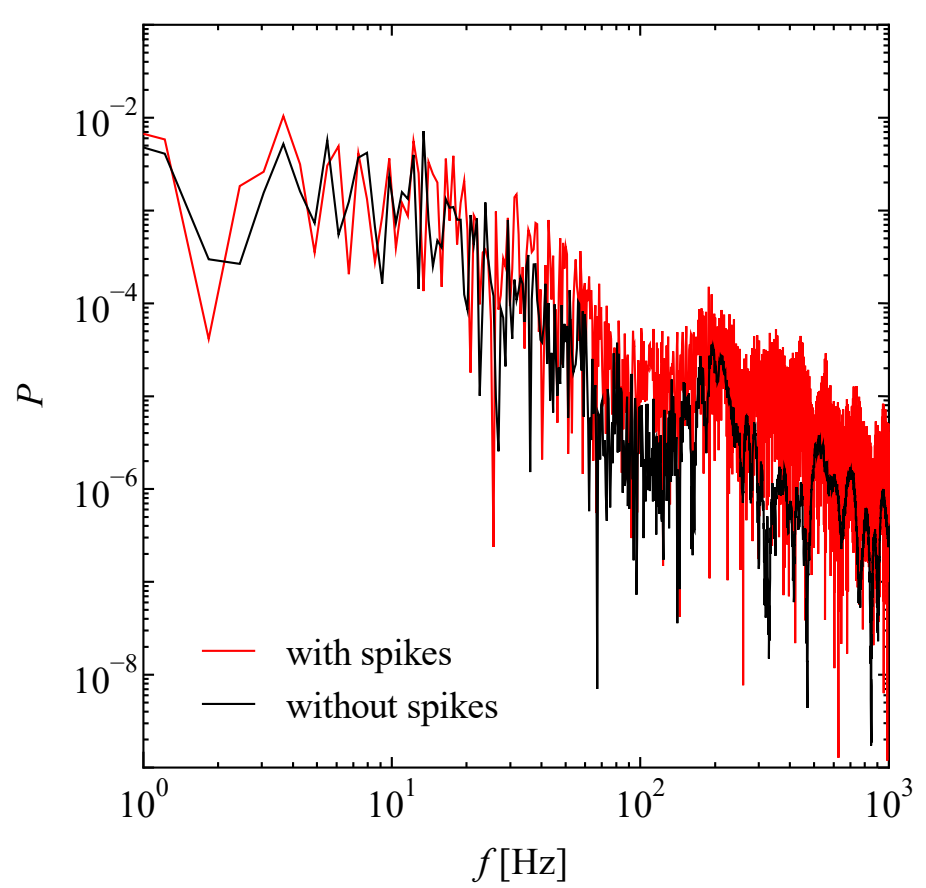

Fig. 13 Comparison of the power spectra of $u$ in Fig.10 for two time ranges, spike-included range of $[0,1.6]$ and spike-excluded range of $[1.6,2.9]$.

height of $\eta=8-9$, which corresponds to $1.6-1.8$ times of the local boundary layer thickness. It also had a long-tapered tail, which trails behind the structure.

Another simultaneous two-point measurement was carried out in order to change the movable probe location from $x=800$ to $700 \mathrm{~mm}$, while keeping the reference probe location at $x=800 \mathrm{~mm}$. The convection velocity of the leading and trailing edges of the detected structure was then estimated from the time delay between the two reference times at two different streamwise stations. The results are plotted in Fig. 12. Here, the velocity at the leading edge, that is, its convection velocity, was approximately $0.82 U_{\infty}-0.92 U_{\infty}$, whereas for the trailing edge this was $0.42 U_{\infty}-0.58 U_{\infty}$. In fact, the local turbulent region grew downstream because of the convection velocity difference, which is consistent with the result of other experimental studies that measured turbulent spots (Grek et al., 1988; Clark et al., 1994; Terashima et al., 2011). Further, these results indicate that the spike waveform was caused by a turbulent spot passing by. However, the number of spikes was only 299 during the sampling time even at $x=900 \mathrm{~mm}$ so that most of time the boundary layer was in the laminar state. This was one reason that the streaky pattern was clearly observed in downstream region, as shown in Fig. 5(a). A comparison of the energy spectra of $u$ in Fig. 10 for two time ranges, $t=0-1.6 \mathrm{~s}$ and $1.3-2.9 \mathrm{~s}$, is presented in Fig. 13. Note that the first range showed the spikes while the latter did not. Moreover, such obvious difference could be observed in the range of several hundred Hz. Furthermore, the waveform in which spikes appeared possessed higher energy in this frequency range. Therefore, it is fair to conclude that the growth of a middle frequency band observed in Fig. 7 was due to the appearance and the increase in turbulent spots.

\section{Conclusions}

In this paper, the response of a boundary layer to localized freestream disturbance was investigated in a wind tunnel experiment. A sheet-type disturbance one-fifth the thickness of the plate was introduced to the right upstream of the plate by ejecting a jet in the downstream direction to compensate the velocity deficit behind the disturbance generator. Consequent, streaky structures similar to an ordinary bypass transition were observed in the boundary layer, although strong disturbances were absent in the freestream outside of the boundary layer. These streaks meandered slowly in the spanwise direction via the streak instability and yielded isolated turbulent regions downstream, referred herein as the turbulent spots. Moreover, the energy growth of the low-frequency band was found to be a result of the meandering motion of the streak, whereas those of the middle- and high-frequency bands of the energy spectrum could be attributed to the appearances of the turbulent spots. 


\section{References}

Andersson, P., Brandt, L., Bottaro, A. and Henningson, S., On the breakdown of boundary layer streaks, Journal of Fluid Mechanics, Vol. 428 (2001), DOI:10.1017/S0022112000002421.

Asai, M., Konishi, Y., Oizumi, Y. and Nishioka, M., Growth and breakdown of low-speed streaks leading to wall turbulence, Journal of Fluid Mechanics, Vol. 586 (2007), DOI:10.1017/S002211200700688X.

Boiko, A. V., Receptivity of a flat plate boundary layer to a free stream axial vortex, European Journal of Mechanics B/Fluids, Vol. 21 (2002), DOI:10.1016/S0997-7546(01)01174-8.

Cherubini, S, Robinet, J. C. and Palma, P. D, Numerical study of the effect of freestream turbulence on by-pass transition in a boundary layer, Energy Procedia., Vol. 45 (2014), DOI:10.1016/j.egypro.2014.01.062.

Clark, J. P., Jones, T. V. and LaGraff, J. E., On the propagation of naturally-occurring turbulent spots, Journal of Engineering Mathematics, Vol. 28, No. 1 (1994), DOI:10.1007/BF02383602.

Eloffson, P. A, Kawakami, M. and Alfredsson, P. H, Experiments on the stability of streamwise streaks in plane Poiseuille flow, Phisics of Fluids., Vol. 11 (1999), DOI:10.1063/1.869962.

Fransson, H. M., Matsubara, M. and Alfredsson, P. H., Transition induced by free-stream turbulence, Journal of Fluid Mechanics, Vol. 527 (2005), DOI:10.1017/S0022112004002770.

Grek, G. R., Kozlov, V. V. and Ramazanov, M. P., Laminar-turbulent transition in the presence of a high level of freestream turbulence, Fluid Dynamics, Vol. 23, No. 6 (1988), DOI:10.1007/BF01051815.

Jacobs, R. G. and Durbin, P. A., Simulation of bypass transition, Journal of Fluid Mechanics, Vol. 428 (2001), pp. $185-212$.

Goit, J. P., Shigeta, M., Izawa, S. and Fukunishi, Y., Prediction of transition point by pursuing local growth of instability waves, Proceedings of annual Meeting of Japan Society of Fluid Mechanics, CD-ROM (2011). (in Japanese).

Matsubara, M. and Alfredsson, P. H., Disturbance growth in boundary layers subjected to free-stream turbulence, Journal of Fluid Mechanics, Vol. 430 (2001), DOI:10.1017/S0022112000002810.

Nagarajan, S., Lele, S. K. and Ferziger, J. H., Leading-edge effects in bypass transition, Journal of Fluid Mechanics, Vol. 572 (2007), DOI:10.1017/S0022112006001893.

Nishio, Y., Shigeta, M., Izawa, S. and Fukunishi, Y., Numerical study on leading-edge receptivity to freestream vertical vorticity, Journal of Fluid Science and Technology, Vol. 8, No. 1 (2013), DOI:10.1299/jfst.8.136.

Reed, H. L., Reshotko, E. and Saric, W. S., Receptivity: the inspiration of Mark Morkovin, 45th AIAA Fluid Dynamics Conference, (2015).

Reshotko, E., Transient growth: a factor in bypass transition, Physics of Fluids, Vol. 13 (2001), DOI:10.1063/1.1358308.

Saric, W. S., Reed, H. L. and Kerschen, E. J., Boundary layer receptivity to freestream disturbances, Annual Review of Fluid Mechanics, Vol. 34 (2002), DOI:10.1146/annurev.fluid.34.082701.161921.

Schlatter, P, Brandt, L., De Lange, H. C. and Henningson D. S., On streak breakdown in bypass transition, Phisics of Fluids., Vol. 20 (2008), DOI:10.1063/1.3005836.

Schrader, L.-U., Brandt, L., Mavriplis, C. and Henningson, D. S., Receptivity to free-stream vorticity of flow past a flat plate with elliptic leading edge, Journal of Fluid Mechanics, Vol. 653 (2010), DOI:10.1017/S0022112010000376.

Terashima, O., Izawa, S., Inasawa, A. and Fukunishi, Y., Streamwise interface of an isolated turbulent region in a laminar flat-plate boundary layer, Transaction of the Japan society of mechanical engineering series B, Vol. 77, No. 773 (2011), pp. 56-65. (in Japanese)

Toda, Y., Morimatsu, M., Nishio, Y. and Ogawa, T., Theoretical model of a flow in a tube with a slit, Proceedings of the AJK 2019 Joint Fluids Engineering Conference, (2019), DOI:10.1115/AJKFluids2019-5257.

Tokugawa, N., Takagi, S., Ueda, Y. and Ido, A., Influence of the external disturbances on natural boundary-layer transition in rectangular wing flows, Journal of Japan Society of Fluid Mechanics, Vol. 24, No. 6 (2005), pp. 629-639. (in Japanese).

Westin, K. J. A., Boiko, A. V., Klingmann, B. G. B., Kozlov, V. V. and Alfredsson, P. H., Experiments in a boundary layer subjected to free stream turbulence, Part 1. Boundary layer structure and receptivity, Journal of Fluid Mechanics, Vol. 281 (1994), DOI:10.1017/S0022112094003083.

Zhigulev, S. V., Uspenskii, A. A. and Ustinov, M. V., Effect of the freestream turbulence scale and the leading edge shape on boundary layer laminar-turbulent transition, Fluid Dynamics, Vol. 44, No. 1 (2009), DOI:10.1134/S0015462809010049. 